\title{
Sonic hedgehog expression in gastric cancer and gastric adenoma
}

\author{
SUN-YOUNG LEE ${ }^{1}$, HYE SEUNG HAN ${ }^{2}$, KYUNG YUNG LEE ${ }^{3}$, TAE SOOK HWANG ${ }^{2}$, \\ JEONG HWAN KIM ${ }^{1}$, IN-KYUNG SUNG ${ }^{1}$, HYUNG-SEOK PARK ${ }^{1}$, \\ CHOON-JO JIN ${ }^{1}$ and KYOO WAN CHOI ${ }^{1}$ \\ Departments of ${ }^{1}$ Internal Medicine, ${ }^{2}$ Pathology and ${ }^{3}$ Surgery, \\ Konkuk University School of Medicine, Seoul, Korea
}

Received November 28, 2006; Accepted February 26, 2007

\begin{abstract}
Hedgehog protein is essential to gastrointestinal tract development, and disruption of the hedgehog signaling pathway is associated with gastrointestinal tumorigenesis. Here, we analyzed the degree of hedgehog expression in gastric cancer and precancerous tissue. From August 2005 to May 2006, 52 gastric cancers and 16 gastric adenomas were obtained from surgically or endoscopically resected specimens. Immunohistochemical staining using sonic hedgehog (Shh) antibody was performed in cancerous and noncancerous tissue portions. Hedgehog expression was assessed based on the summed scores of the intensity and proportion of Shh staining. According to Lauren's classification, Shh expression was stronger in the intestinal type than in the diffuse type $(\mathrm{p}<0.001)$. Although Shh expression was not related to the location, size, metastatic status, or mucin phenotype of the gastric cancer, the expression was stronger in the tubular type of gastric cancer than in the mucinous and signet-ring cell types $(p=0.001)$. Shh expression was stronger in gastric adenoma than in the diffuse-type gastric cancer $(\mathrm{p}<0.001)$, but revealed no difference with that of the intestinal-type gastric cancer $(p=0.30)$. Shh expression was strongest in all types of intestinal metaplasia of noncancerous tissues. Shh expression is related to the intestinal type of gastric cancer. The stronger Shh expression in intestinal metaplasia and gastric adenoma indicates that hedgehog protein is involved at an early phase of gastric carcinogenesis.
\end{abstract}

\section{Introduction}

Gastric cancer is one of the most frequent malignant tumors in Korea, contributing significantly to cancer mortality (1). It

Correspondence to: Dr Hye Seung Han, Department of Pathology, Konkuk University School of Medicine, 4-12 Hwayangdong, Gwangjin-gu, Seoul 143-729, Korea

E-mail: aphsh@kuh.ac.kr

Key words: gastric cancer, adenoma, intestinal type, diffuse type, hedgehog is clear that gastric carcinogenesis is a multifactorial and multistep process requiring sequential alterations in genes codifying for tumor suppressors, proto-oncogenes, gatekeeper genes, enzymes, growth factors, and membrane or nuclear receptors (the so-called multihit hypothesis) (2). Among these, recent attention has focused on sonic hedgehog (Shh), which is reportedly elevated in human gastric cancer $(3,4)$.

The hedgehog signaling pathway is critical to normal gastrointestinal development and function, and the stomach is one of the largest sources of Shh protein (5). Shh is an important endodermal signal in endodermal mesenchymal interactions during development of the gut in vertebrates, especially in the gastric gland (6). Although previous studies have found that Shh expression varies with the type of gastric cancer (7-10), the role of Shh in gastric tumorigenesis remains unclear. Therefore, we analyzed Shh expression in gastric cancer and precancerous lesions such as gastric adenoma and intestinal metaplasia.

\section{Materials and methods}

Materials. From August 2005 to May 2006, 50 patients (with 52 separate gastric cancers and 5 gastric adenomas) who underwent gastrectomy for gastric cancer, and 11 patients (with 11 gastric adenomas) who underwent endoscopic resection for gastric adenoma in Konkuk University Hospital were enrolled in the study. A total of 52 gastric cancers and 16 adenomas were obtained from the patients since 5 had two coexisting neoplasms and 1 patient had three coexisting lesions in the same stomach. All of the patients provided written informed consent prior to undergoing the procedure. This prospective study was approved by the institutional review board of Konkuk University School of Medicine, which confirmed that the study was in accordance with the ethical guidelines of The Helsinki Declaration.

A total of 68 tissue-array blocks were obtained from the 16 cases of gastric adenoma specimens and 52 cases of formalin-fixed, paraffin-embedded gastric cancer tissues without visible tumor necrosis. Adjacent paired normal gastric tissues of the antrum and corpus were also studied. Tissue samples showing intestinal metaplasia were obtained from the 35 antral mucosa and 29 body mucosa apart from the gastric cancer. 
Immunohistochemical staining. Serial 4- $\mu$ m-thick sections were cut from formalin-fixed and paraffin-embedded tissues and mounted on glass slides coated with silane (Matsunami, Tokyo, Japan), deparaffinized in xylene, dehydrated in ethanol, and dried in a microwave oven with $10 \mathrm{mM}$ Na-citrate buffer (pH 6.0) for antigen retrieval. Immunohistochemical staining for Shh was performed using the Envision kit (Dako, Glostrup, Denmark). Endogenous peroxidase activities were blocked with $3 \%$ hydrogen peroxide in methanol for $20 \mathrm{~min}$. After nonspecific binding was blocked, sections were incubated with rabbit polyclonal Shh antibody $(1: 100$, Santa Cruz, CA, USA), as primary antibodies, at room temperature for $1 \mathrm{~h}$. After washing with PBS three times, the sections were incubated with the enzyme-conjugated secondary antibody. The sections were incubated with the DAB substrate for less than $3 \mathrm{~min}$, and hematoxylin was used for counterstaining.

To classify the gastric carcinomas according to the mucin phenotype, immunohistochemical staining with the primary antibodies: MUC5AC (45M/1, 1:2000), MUC6 (MCN6.01, 1:200), MUC2 (996/1, 1:2000) and CD10 (56C6, 1:50) (all from Neomarker, CA, USA) was performed using the iVIEW DAB detection kit and Benchmark XT (both from Ventana Medical Systems, Arizona, USA). Negative controls were performed in all cases by omitting the primary antibodies.

Analysis of expression status. Expression status was quantified by scoring both the intensity and proportion of Shh staining. The intensity of the staining was scored as $0,1,2$, or 3 , and the proportion of the expression in the tissue was scored as 0 $(0-5 \%), 1(6-33 \%), 2(34-66 \%)$, or 3 (67-100\%). The summed scores of intensity and proportion were quantified as negative (score of 0 ), weakly positive (1-2 points), moderately positive (3-4 points), or strongly positive (5-6 points) for each lesion.

The gastric cancers were subclassified as the gastric or intestinal mucin phenotype if $>10 \%$ of cancer cells exhibited gastric (MUC5AC and/or MUC6) or intestinal (MUC2 or CD10) markers, respectively. Tumors were classified as gastric and intestinal mixed type when $>10 \%$ of neoplastic cells showed both gastric and intestinal markers and as unclassified when $<10 \%$ of neoplastic cells showed either gastric or intestinal markers.

Statistical analysis. A p value $<0.05$ was considered statistically significant. Differences between the groups were analyzed using the Chi-square and t-tests. Age and size data were expressed as the mean $\pm \mathrm{SE}$ of the values.

\section{Results}

Characteristics of the tumors. Fifty-two gastric cancers were present in 36 men and 16 women aged $62.4 \pm 12.0$ years, and 16 gastric adenomas were present in 13 men and 3 women aged $63.8 \pm 7.4$ years. The location of the gastric cancer was the antrum in 22 cases, corpus in 24 , and other in 6 , while that of the gastric adenoma was the antrum in 11 cases, corpus in 4 , and other in 1 case. The sizes of the gastric cancers and adenomas were $25.7 \pm 29.8$ and $0.8 \pm 7.4 \mathrm{~cm}^{2}$, respectively.

Of the 52 gastric cancers (24 early and 28 advanced), 41 cases were tubular type, 4 were mucinous type, and 7 were signet-ring cell type. According to Lauren's classification (11), 37 cases were intestinal type and 15 were diffuse type. In terms of the mucin phenotype, 12 cases were gastric type, 19 were intestinal type, 13 were mixed type, and 8 were unclassified. Lymph node metastasis was evident in 23 cases $(44.2 \%)$. Lymphatic invasion was evident in 24 cases $(46.2 \%)$, venous invasion in $4(7.7 \%)$, and neural invasion in $9(17.3 \%)$.

Shh expression in noncancerous tissue. Of the 16 gastric adenomas, the Shh expression was strongly positive (5-6 points) in 15 cases and moderately positive (4 points) in 1 case. In normal gastric tissue, Shh expression was near to completely negative in the upper region of the normal gastric epithelium and weakly positive in the lower part containing mucus neck cells and glandular cells. In contrast, Shh expression was strongly positive in the parietal cells of the fundic glands. Adjacent normal gastric tissues showing intestinal metaplasia (24 complete type and 40 incomplete) which were obtained adjacent to the gastric cancer exhibited the strongest Shh expression in all 64 cases (Fig. 1).

Shh expression in cancerous tissue. The status of Shh expression in gastric cancers is summarized in Table I. The Shh expression was stronger in intestinal- than diffuse-type gastric cancer $(\mathrm{p}<0.001)$ (Fig. 2). When compared with that of the gastric adenoma, Shh expression was stronger in gastric adenoma than in the diffuse-type gastric cancer $(\mathrm{p}<0.001)$, but revealed no difference with that of the intestinal-type gastric cancer $(\mathrm{p}=0.30)$ (Fig. 3).

\section{Discussion}

The hedgehog signaling pathway controls cell proliferation via cell cycle regulation, and hence also tissue regeneration and carcinogenesis in gastric cancer (12). In the present study, Shh expression was stronger in the precancerous lesions such as intestinal metaplasia and gastric adenoma as well as in intestinal-type gastric cancer but not in diffuse-type gastric cancer. This finding indicates that hedgehog activation is related to the early phase of gastric carcinogenesis in intestinal-type gastric cancer.

Intestinal metaplasia has been considered a precancerous lesion of intestinal-type gastric carcinoma (13). Intestinal-type adenocarcinomas are usually located in the distal stomach and have a glandular structure that is believed to arise from an intestinal metaplastic epithelium, and is thought to develop through sequential changes; chronic superficial gastritis, chronic atrophic gastritis, intestinal metaplasia, and dysplasia (14). In contrast, diffuse-type adenocarcinomas principally invade the cardia and exhibit a poor grandular formation attributed to gastric mucous cells thought to arise from hyperplastic stem or precursor cells (15). In the present study, Shh expression was stronger in intestinal- than in diffuse-type gastric cancer. Our findings suggest that these two types have different backgrounds in Shh expression, and that the close relationship between Shh and intestinal-type gastric cancer represents a genetic model common to gastric cancer.

In terms of the pathological classification of gastric cancer, Shh expression was higher in the tubular type than in the 

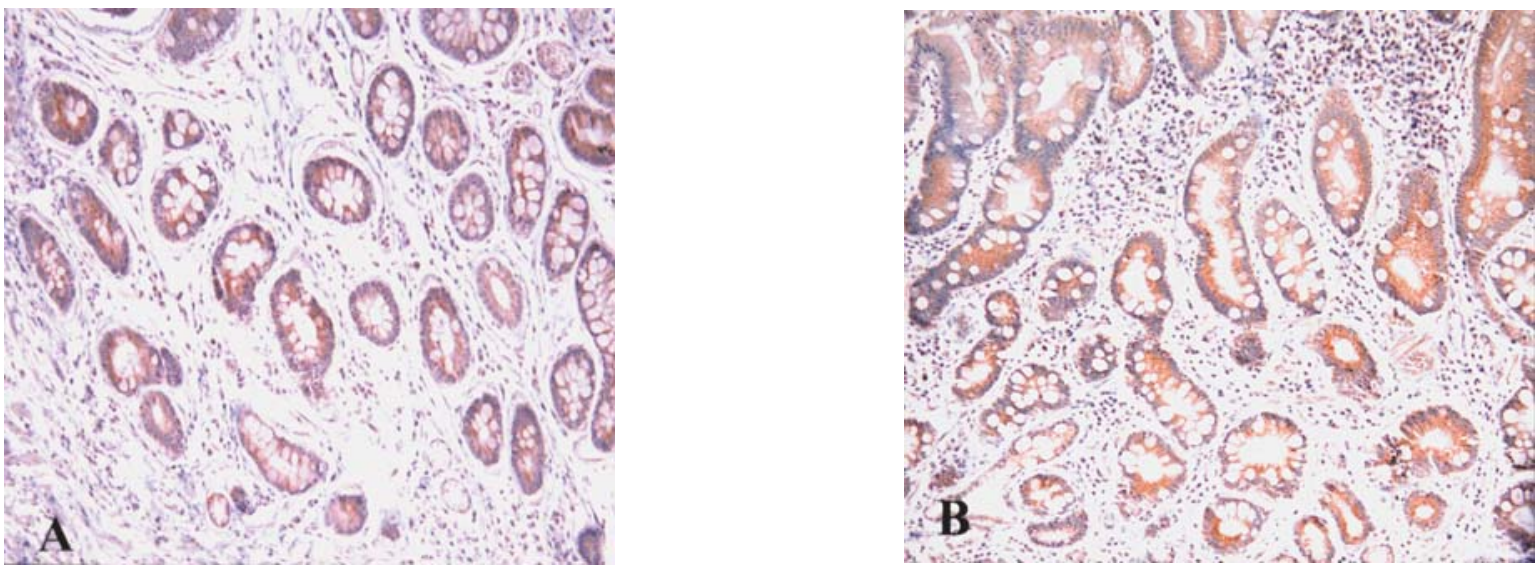

Figure 1. Immunohistochemical staining of sonic hedgehog (Shh) in intestinal metaplasia. All of the 64 adjacent noncancerous tissue samples showing intestinal metaplasia revealed a high Shh expression score (5 or 6 points) irrespective of the type of intestinal metaplasia. (A) Complete type. Shh staining revealed strong expression (immunohistochemical staining x200). (B) Incomplete type. Shh staining revealed strong expression (immunohistochemical staining $\mathrm{x} 200)$.
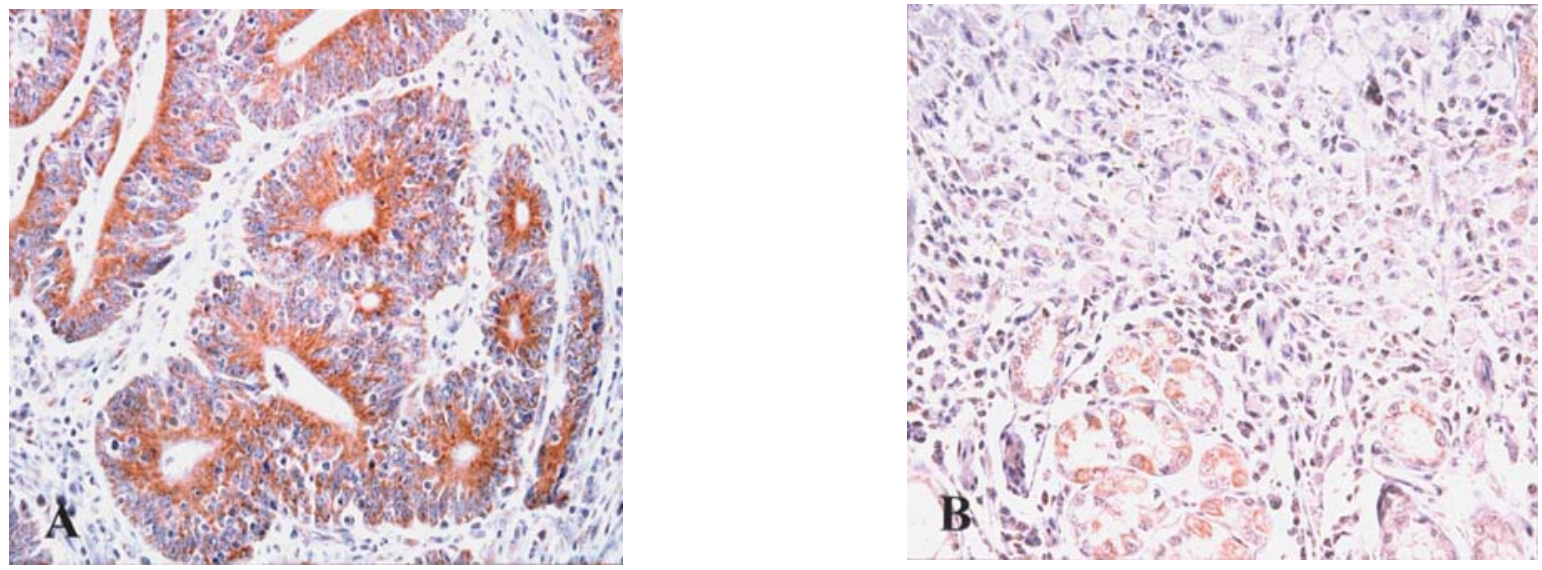

Figure 2. Immunohistochemical staining of sonic hedgehog (Shh) in gastric cancer. According to Lauren's classification, the Shh expression was stronger in the intestinal type than in the diffuse type $(\mathrm{p}<0.001)$. (A) Intestinal type. Shh staining revealed strong expression in cancer cells (immunohistochemical staining $\mathrm{x} 400$ ). (B) Diffuse type. Shh staining revealed weak expression in a few of the cancer cells (immunohistochemical staining $\mathrm{x} 400$ ).

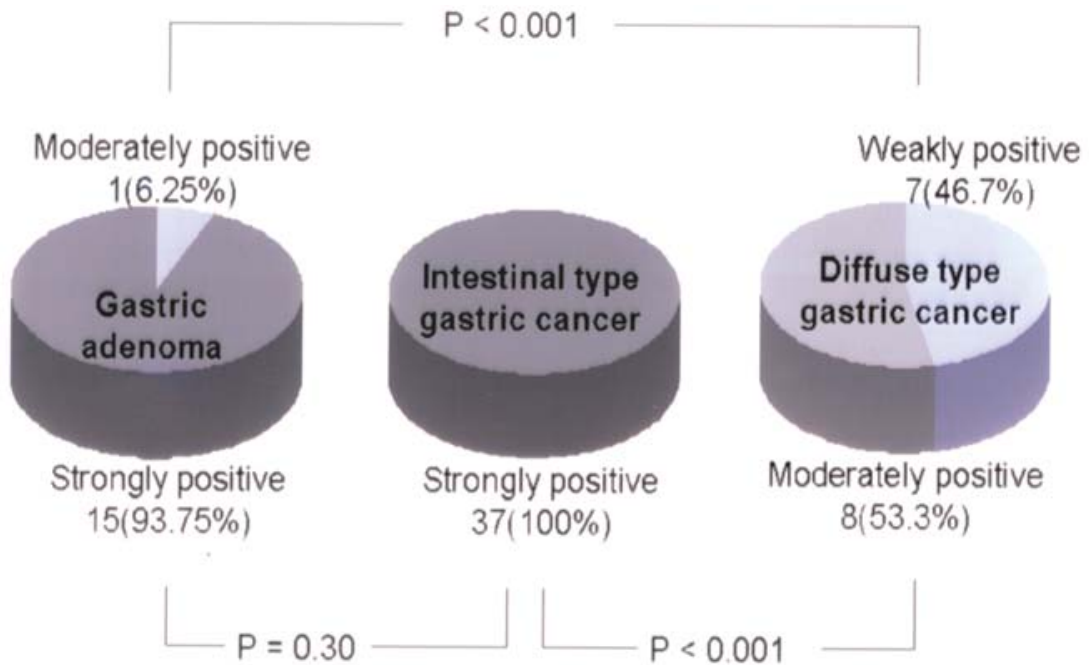

Figure 3. Comparison of sonic hedgehog (Shh) expression between gastric adenoma, intestinal-type gastric cancer, and diffuse-type gastric cancer. Shh expression was quantified as weakly positive (1-2 points), moderately positive (3-4 points), or strongly positive (5-6 points). Shh expression was stronger in gastric adenoma than in the diffuse-type gastric cancer $(\mathrm{p}<0.001)$, but revealed no difference with that of the intestinal-type gastric cancer $(\mathrm{p}=0.30)$. 
Table I. Sonic hedgehog expression in gastric cancers.

\begin{tabular}{|c|c|c|c|c|}
\hline & \multicolumn{3}{|c|}{ Sonic hedgehog expression ${ }^{a}$} & \multirow[b]{2}{*}{$\mathrm{p}$ value } \\
\hline & $\begin{array}{l}\text { Weakly positive } \\
\qquad(\mathrm{n}=7)\end{array}$ & $\begin{array}{l}\text { Moderately positive } \\
\qquad(\mathrm{n}=8)\end{array}$ & $\begin{array}{l}\text { Strongly positive } \\
\qquad(\mathrm{n}=37)\end{array}$ & \\
\hline Lauren's classification & & & & $<0.001$ \\
\hline Intestinal & 0 & 0 & 37 & \\
\hline Diffuse & 7 & 8 & 0 & \\
\hline Type of carcinoma & & & & 0.001 \\
\hline Tubular & 2 & 5 & 34 & \\
\hline Mucinous & 1 & 1 & 2 & \\
\hline Signet-ring cell & 4 & 2 & 1 & \\
\hline Mucin phenotype & & & & NS \\
\hline Unclassified & 0 & 1 & 7 & \\
\hline Gastric & 4 & 2 & 6 & \\
\hline Intestinal & 2 & 1 & 16 & \\
\hline Mixed & 1 & 4 & 8 & \\
\hline Location & & & & NS \\
\hline Antrum & 3 & 4 & 15 & \\
\hline Corpus & 4 & 3 & 17 & \\
\hline Others & 0 & 1 & 5 & \\
\hline Size $\left(\mathrm{cm}^{2}\right.$, mean $\left.\pm \mathrm{SD}\right)$ & $28.3 \pm 34.9$ & $35.7 \pm 33.3$ & $23.1 \pm 28.4$ & NS \\
\hline $\begin{array}{l}\text { Presence of lymph node } \\
\text { metastasis }(\%)\end{array}$ & $4(57.1 \%)$ & $3(37.5 \%)$ & $16(43.2 \%)$ & NS \\
\hline
\end{tabular}

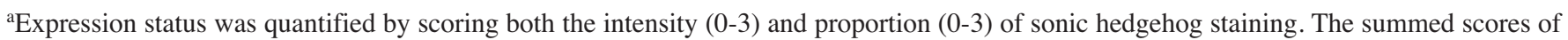
intensity and proportion were quantified as weakly positive (1-2 points), moderately positive (3-4 points), or strongly positive (5-6 points) for each lesion.

mucinous and signet-ring cell types. Although Shh expression is reportedly associated with clinical stage and tumor invasion $(8,9)$, we found that Shh expression was not related to the location, size, metastatic status, or mucin phenotype of the gastric cancer. These contrasting results on Shh expression may reflect ethnic, racial, or geographic differences, discrepancies due to different methods in assessing Shh expression, or the small number of subjects in our study or in other studies.

In conclusion, stronger Shh expression in intestinal metaplasia and gastric adenoma indicates that hedgehog protein is involved at an early phase of gastric tumorigenesis. Based on these results, Shh screening and the precise selection of therapeutic options should be based on the type of gastric cancer, and antihedgehog signaling could be used to detect gastric tumorigenesis at an earlier stage especially in the intestinal-type gastric cancer.

\section{Acknowledgements}

This study was supported by the BK 21 project of Konkuk University, 2006.

\section{References}

1. Korean Central Cancer Registry 2005: Korean Ministry of Health and Welfare. Annual Report of the Korean Central Cancer Registry, Seoul, 2005.

2. Seregni E, Ferrari L, Martinetti A and Bombardieri E: Diagnostic and prognostic tumor markers in the gastrointestinal tract. Semin Surg Oncol 20: 147-166, 2001.

3. Berman DM, Karhadkar SS, Maitra A, et al: Widespread requirement for Hedgehog ligand stimulation in growth of digestive tract tumours. Nature 425: 846-851, 2003.

4. Thayer SP, di Magliano MP, Heiser PW, et al: Hedgehog is an early and late mediator of pancreatic cancer tumorigenesis. Nature 425: 851-856, 2003.

5. Ramalho-Santos M, Melton DA and McMahon AP: Hedgehog signals regulate multiple aspects of gastrointestinal development. Development 127: 2763-2772, 2000.

6. van den Brink GR, Hardwick JC, Nielsen C, et al: Sonic hedgehog expression correlates with fundic gland differentiation in the gastrointestinal tract. Gut 51: 628-633, 2002.

7. Fukaya M, Isohata N, Ohta $\mathrm{H}$, et al: Hedgehog signal activation in gastric pit cells and in diffuse-type gastric cancer. Gastroenterology 131: 14-29, 2006.

8. Wang LH, Choi YL, Hua XY, et al: Increased expression of sonic hedgehog and altered methylation of its promoter region in gastric cancer and its related lesions. Mod Pathol 19: 675-683, 2006.

9. Ma X, Chen K, Huang S, et al: Frequent activation of the hedgehog pathway in advanced gastric adenocarcinomas. Carcinogenesis 26: 1698-1705, 2005. 
10. Shiotani A, Iishi H, Uedo N, et al: Evidence that loss of sonic hedgehog is an indicator of Helicobacter pylori-induced atrophic gastritis progressing to gastric cancer. Am J Gastroenterol 100: 581-587, 2005.

11. Lauren P: The two histological main types of gastric carcinoma: Diffuse- and so-called intestinal-type carcinoma - an attempt at a histoclinical classification. Acta Pathol Microbiol Scand 64: 31-49, 1965.

12. Katoh $\mathrm{Y}$ and Katoh M: Hedgehog signaling pathway and gastric cancer. Cancer Biol Ther 4: 1050-1054, 2005.
13. Correa $\mathrm{P}$ and Shiano YH: Phenotypic and genotypic events in gastric carcinogenesis. Cancer Res 54: S1941-S1943, 1994.

14. Yuasa Y: Control of gut differentiation and intestinal type gastric carcinogenesis. Nat Rev Cancer 3: 592-600, 2003.

15. Si-Chun M: Cellular and molecular pathology of gastric carcinoma and precursor lesions: a critical review. Gastric Cancer 1: 31-50, 1998. 